\title{
Influenza vaccine
}

\section{A Pai, B V Pai}

\section{Where do we stand?}

n the handbook Immunisation against infectious diseases (Green Book) published by HMSO in 1996, ${ }^{1}$ influenza vaccine has been recommended for the following categories in children:

- Chronic cardiorespiratory conditions, including asthma

- Diabetes

- Chronic kidney problems

- Diseases or treatments affecting the immune system

- Chronic debilitating conditions.

The reason for giving influenza vaccine is that influenza can cause considerable morbidity and mortality in the above mentioned categories. According to the Public Health Laboratory website, influenza vaccine uptake is $10-12 \%$ in the $<65$ year age group (no separate figure for the paediatric age group).

Diabetes and asthma form a large part of paediatric practice, and the incidence of both these disorders is increasing. Despite this, uptake of this vaccine is extremely poor in these two categories.

When going through the British Thoracic Guidelines on Asthma for children, one fails to come across any mention of influenza vaccine in the long term management of asthma. Looking at the guidelines published for Diabetes and Haemato-oncological conditions, influenza vaccine does not find a place.

These findings are not in keeping with the official recommendations, as published in the Green Book. To understand this we scanned the current evidence, concentrating on three aspects: safety, efficacy, and benefit:risk ratio.

"Inactivated influenza vaccine is safe in children"
There are many studies looking at the safety of inactivated influenza vaccine (the currently available vaccine in the UK). ${ }^{23}$ Initial reports suggested that influenza vaccine may exacerbate asthma or reduce lung function. The Cochrane systematic review group looked at this aspect closely and found no evidence to support this. ${ }^{4}$ On current evidence, it is safe to say that inactivated influenza vaccine is safe in children.

"Efficacy of influenza is well proven in children"

Efficacy has been well proven, and with two doses of influenza vaccine the uptake is around $94 \%$. This uptake is similar in cancer patients undergoing treatment. $^{5}$

"Risk: benefit ratio of influenza vaccine is doubtful in the paediatric population"

Risk:benefit ratio is a contentious issue. The Cochrane review group which looked at vaccines for prevention of influenza in people with asthma concluded that there is insufficient evidence to assess benefit and risk of influenza vaccination. A study by Johnston et al on the role of viruses in exacerbation of asthma suggested that $80 \%$ of acute exacerbation are viral infection related, of which the contribution of rhinoviruses was $80 \%{ }^{6}$ A study from the Netherlands concluded that influenza vaccine is effective in preventing acute exacerbation in $55 \%$ of asthmatic patient below 6 years of age compared to 5\% of asthmatics above 6 years. ${ }^{7}$ There are no studies on the effectiveness of influenza vaccine in diabetes.

In an overcrowded immunisation schedule, with recent controversy over
MMR vaccine, it is difficult for general practitioners and paediatricians to recommend influenza vaccine in the absence of strong clinical evidence to parents of asthmatic and diabetic children. A recent paper from the USA suggests that uptake of influenza vaccine is barely $10 \%$ in the asthmatic population, despite the availability of vaccine in nasal spray form. ${ }^{8}$ This suggests that doctors themselves are not convinced of the role of influenza vaccine in asthma and diabetes.

With this background, should the Green Book recommend routine influenza vaccination for diabetic and asthmatic children?

Arch Dis Child 2003;88:665

\section{Authors' affiliations}

V A Pai, B V Pai, Royal Manchester Children's Hospital; St James University Hospital, Leeds, UK

Correspondence to: Dr V A Pai, 34 Thorne Lane, Roundhay, Leeds LS8 INF, UK; binapai@hotmail.com

\section{REFERENCES}

1 HMSO. Immunization against infectious diseases. "The Green Book". London: HMSO 1996

2 Neuzil KM. Safety of inactivated influenza vaccine in adult and children with asthma. J Paediatr 2002;140:632

3 Gonzales M, Pirez MC. Safety and immunogenecity of paediatric preparation of influenza vaccine. Arch Dis Child 2000;83:488-91.

4 Cochrane review. Vaccine for preventing influenza in people with asthma. Cochrane Library, Issue 3, 2002.

5 Chisholm JC, Devine T, Charlett A, et al. Response to influenza immunization during treatment for cancer. Arch Dis Child treatment for cancer

6 Johnston SL, Pattemore PK, Sanderson G, et al. Community study role of viral infection in exacerbation of asthma in 9-11 yr old children. BM 1995;310:1225-8.

7 Smit AJ. Clinical effectiveness of conventional influenza vaccination in asthma. Epidemiol Infect 2002; 128:205-1 1.

8 Gaglani MJ. Rational and approach to target children with asthma for annual influenza vaccine. Semin Paediatr Infect Dis 2002; 13:97-103.

9 Beyer WE. Routine influenza vaccination for healthy children, old concept-new technology. Arch Dis Child 2000;83:461. 